\title{
La résistance aux antituberculeux au Canada : 2018
}

\section{Marie LaFreniere ${ }^{1}$, Demy Dam ${ }^{1,2}$, Réseau technique canadien de laboratoires du tuberculose ${ }^{3}$, Lori Strudwick ${ }^{4}$, Sarah McDermott ${ }^{1}$}

\section{Résumé}

Introduction : La tuberculose pharmaco-résistante est une question de santé publique d'importance mondiale constituant une menace contre les efforts entrepris pour lutter contre la tuberculose. Le Canada effectue une surveillance l'échelle nationale pour détecter les tendances de la pharmaco-résistance et pour documenter les progrès réalisés en vue de l'élimination de la tuberculose.

Objectif : Décrire les tendances de la résistance aux antituberculeux au Canada de 2008 à 2018, par répartition géographique et caractéristiques démographiques, en mettant l'accent sur 2018.

Méthodes : Les données sur la résistance aux antituberculeux sont recueillies à travers deux systèmes de surveillance indépendants gérés par l'Agence de la santé publique du Canada : le Système canadien de surveillance en laboratoire de la tuberculose (SCSLT) et le Système canadien de déclaration des cas de tuberculose (SCDTB). Les données provenant de ces systèmes ont été analysées et des statistiques descriptives ont été présentées par profil de résistance, lieu de résidence (province), groupes d'âge, sexe et pays de naissance.

Résultats : En 2018, le test de sensibilité aux antituberculeux a été réalisé sur 1459 isolats de tuberculose; soit une baisse de 4,3\% par rapport à 2017. La résistance à tout antituberculeux de première intention a été signalée dans 148 isolats (10,1\%), comparativement à $123(8,1 \%)$ en 2017. Parmi ceux-ci, 121 étaient monorésistants, cinq étaient polyrésistants, 21 étaient multirésistants (TB-MR) et un seul était ultrarésistant (TB-UR). La résistance aux antituberculeux a été signalée dans toutes les provinces et tous les territoires, à l'exception de l'Île-du-Prince-Édouard, des Territoiresdu-Nord-Ouest et du Yukon. Chez les personnes de moins de 15 ans, très peu de résistance aux antituberculeux a été constatée. Chez les personnes de 15 ans et plus, la distribution de la résistance aux antituberculeux variait sans aucune tendance discernable. La proportion de la résistance aux antituberculeux était légèrement plus élevée chez les femmes que chez les hommes. On constate que $10,7 \%$ des cas de tuberculose déclarés entre 2006 et 2016 chez des personnes nées à l'étranger étaient résistants aux antituberculeux. Parmi les cas de tuberculose des personnes nées au Canada, la résistance aux antituberculeux était de $9,3 \%$ chez les non Autochtones, contre $2,4 \%$ chez les Autochtones.

Conclusion : En 2018, la proportion d'isolats présentant une résistance aux antituberculeux au Canada est demeurée faible et inférieure aux moyennes internationales; avec des tendances stables de la résistance aux antituberculeux, tant géographiques que démographiques.
Cette oeuvre est mise à la disposition selon les termes de la licence internationale Creative Commons Attribution 4.0

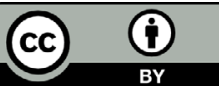

Affiliations

${ }^{1}$ Le Centre de la lutte contre les maladies transmissibles et les infections, l'Agence de la Santé publique Canada,

Ottawa, ON

${ }^{2}$ Faculté de médecine, Département d'épidémiologie, de biostatistique et de santé au travail, Université McGill, Montréal, QC

${ }^{3}$ Réseau technique canadien de laboratoires de tuberculose

${ }^{4}$ Gouvernement du Yukon, Ministère de la Santé et des Affaires sociales, Centre de lutte contre les maladies transmissibles du Yukon, Whitehorse, YK

Remarque: La composition du réseau est précisée à la section Collaborateurs à la fin de l'article

${ }^{\star}$ Correspondance : phac.

tb.surveillance.aspc@canada.ca

Citation proposée : LaFreniere M, Dam D, Réseau technique canadien de laboratoires de tuberculose, Strudwick L, McDermott S. Résistance aux antituberculeux au Canada : 2018. Relevé des maladies transmissibles au

Canada 2020;46(1):10-6. https://doi.org/10.14745/ccdr.v46i01a02f

Mots-clés : tuberculose, surveillance, résistance aux antituberculeux, Canada

\section{Introduction}

La tuberculose (TB), une maladie infectieuse transmissible par voie aérienne et causée par la bactérie Mycobacterium tuberculosis, demeure une cause majeure de maladie à l'échelle mondiale. L'Organisation mondiale de la santé (OMS) a estimé que 10 millions de cas ont été diagnostiqués en 2017. On considère que la tuberculose est la première cause infectieuse 
de décès dans le monde (1). Même s'il existe des traitements efficaces, la lutte risque d'être entravée par l'émergence d'une résistance aux antituberculeux. Les souches de tuberculose résistantes aux antituberculeux de première intention pourraient prendre beaucoup plus de temps à traiter à l'aide de médicaments aux effets secondaires plus graves (2). Selon l'OMS, environ 558000 cas de tuberculose pharmaco-résistante à l'antituberculeux de première intention, la rifampicine, ont été diagnostiqués en 2017; de ce nombre, 82,0 \% étaient des cas de tuberculose multirésistante (TB-MR). Parmi les cas de tuberculose multirésistante diagnostiqués, environ 8,5\% étaient ultrarésistants (TB-UR) (1).

En septembre 2018, I'Assemblée générale des Nations unies a tenu la première réunion de haut niveau sur la tuberculose. Lors de cette réunion, les États membres ont réaffirmé leur engagement à mettre fin à l'épidémie mondiale de tuberculose d'ici 2030 (3). Créée dans la foulée de cette réunion, la Déclaration politique reconnaît que la tuberculose a des répercussions importantes sur la santé mondiale et que si cette question n'est pas adéquatement traitée, les progrès réalisés pour l'élimination de la tuberculose pourraient être réduits à néant (4). Même si la proportion des isolats de tuberculose pharmaco-résistante au Canada est restée faible au cours de la précédente décennie (5), il est important de continuer la surveillance épidémiologique de la tuberculose pharmacorésistante pour monitorer l'évolution de la résistance aux antituberculeux au Canada, et informer les responsables de la santé publique sur les mesures appropriées à prendre. En outre, les données permettent de surveiller les avancées du Canada sur I'élimination de la tuberculose et de mesurer le fardeau mondial que représente la tuberculose pharmaco-résistante.

Les Normes canadiennes pour la lutte antituberculeuse recommandent que tous les isolats de tuberculose à culture positive au Canada subissent des tests de sensibilité aux antituberculeux afin de déterminer le meilleur traitement antituberculeux à suivre (2). Ces données peuvent également servir à la surveillance de la résistance aux antituberculeux. Le Système canadien de surveillance en laboratoire de la tuberculose (SCSLT) a été créé en 1998 pour surveiller les tendances et les profils émergents de la résistance aux antituberculeux au Canada (6). Le Système canadien de déclaration des cas de tuberculose (SCDTB) quant à lui, conserve des données démographiques et cliniques non nominales sur les cas de tuberculose active diagnostiqués au Canada.

L'objectif du présent article est de décrire les tendances de la résistance aux antituberculeux au Canada de 2008 à 2018, en mettant l'accent sur 2018, ainsi que les tendances géographiques et démographiques.

\section{Méthodes}

\section{Source des données}

Les données ont été tirées et analysées à partir de deux systèmes de surveillance, le SCSLT et le SCDTB.

Le SCSLT est un système de surveillance des laboratoires fondé sur les isolats et utilisé pour surveiller la résistance aux antituberculeux partout au Canada. (Pour connaître les définitions des profils de résistance aux antituberculeux, consultez le tableau 1). Chaque année, les laboratoires provinciaux de tuberculose soumettent volontairement les résultats des tests phénotypiques de sensibilité aux antituberculeux effectués l'année précédente sur des isolats provenant de cas confirmés par culture de tuberculose. Des données démographiques de base non nominales (sexe, âge et province ou territoire de résidence) sont également recueillies. Des détails additionnels sur la méthodologie de collecte des données du SCSLT, la gestion des données et d'autres processus de laboratoire ont précédemment été présentés (6). Les données couvertes dans le présent article s'étendent jusqu'en 2018 inclusivement.

\section{Tableau 1 : Définitions des profils de résistance aux antituberculeux}

\begin{tabular}{|l|l|}
\hline \multicolumn{1}{|c|}{ Type de résistance } & \multicolumn{1}{c|}{ Définition } \\
\hline Monorésistance & $\begin{array}{l}\text { Résistance à un seul antituberculeux de } \\
\text { première intention (isoniazide, rifampicine, } \\
\text { éthambutol ou pyrazinamide) }\end{array}$ \\
\hline Polyrésistance & $\begin{array}{l}\text { Résistance à plus d'un antituberculeux } \\
\text { de première intention, à l'exception de la } \\
\text { combinaison isoniazide et rifampicine }\end{array}$ \\
\hline $\begin{array}{l}\text { Tuberculose } \\
\text { multirésistante (TB-MR) }\end{array}$ & $\begin{array}{l}\text { Résistance à l'isoniazide ET à la rifampicine } \\
\text { avec ou sans résistance à d'autres } \\
\text { antituberculeux }\end{array}$ \\
\hline $\begin{array}{l}\text { Tuberculose } \\
\text { ultrarésistante (TB-UR) }\end{array}$ & $\begin{array}{l}\text { Résistance à l'isoniazide ET à la rifampicine } \\
\text { ET à toute fluoroquinolone ET à au moins } \\
\text { un des trois antituberculeux injectables } \\
\text { de deuxième intention (amikacine, } \\
\text { capréomycine ou kanamycine) }\end{array}$ \\
\hline
\end{tabular}

Tout isolat de tuberculose ayant démontré des cultures positives au complexe M. tuberculosis ( $M$. tuberculosis, M. africanum, M. canetti, M. caprae, M. microti, M. pinnipedii ou $M$. bovis) a été inclus dans les analyses. Les isolats positifs pour $M$. bovis Bacillus Calmette-Guérin (BCG) ont été exclus, car ils représentent une complication de la vaccination contre la tuberculose souvent observée chez les patients immunodéprimés et, cette souche n'est pas infectieuse.

Alors que le SCSLT collecte des données sur les isolats de M. tuberculosis, le SCDTB est un système de surveillance fondé sur des cas de tuberculose active au Canada, y compris les cas ayant nécessité de nouveaux traitements. Le SCDTB collecte des données sur la résistance aux antituberculeux et les institutions 
sanitaires provinciales et territoriales se chargent de les rapporter à l'Agence de la santé publique du Canada.

Dans le présent article, les chercheurs utilisent les données du SCDTB pour décrire la résistance aux antituberculeux par pays de naissance; avec les Canadiens de naissance stratifiés davantage en Autochtones et non Autochtones. Des renseignements détaillés sur le SCDTB ont été précédemment présentés (7). Les données du SCDTB couvertes dans le présent article s'étendent jusqu'en 2016 inclusivement.

\section{Analyse des données}

Dans la mesure du possible, les doublons potentiels ont été identifiés à partir des paramètres démographiques suivants : sexe, date de naissance ou âge, et province ou territoire. Des clarifications appropriées ont été obtenues auprès des laboratoires provinciaux correspondants sur des doublons potentiels, ainsi que sur toute donnée manquante. Après la tabulation des données, les totaux des isolats ont été envoyés aux provinces et territoires pour confirmation de leur exactitude. Les logiciels SAS Enterprise Guide 5.1 (Cary (Caroline du Nord, États-Unis)) et Microsoft Excel 2010 (Redmond (Washington, États-Unis)) ont été utilisés pour le nettoyage et l'analyse des données.

Des statistiques descriptives sur les données, par profils géographiques, démographiques et de résistance aux antituberculeux, ont été calculées et comparées aux tendances des 10 dernières années. Aucune méthodologie statistique n'a été utilisée pour l'analyse comparative. Des tableaux de données supplémentaires sont disponibles sur demande (se référer à I'annexe pour la liste des tableaux).

\section{Résultats}

\section{Profils de résistance aux antituberculeux en 2018}

En 2018, 1475 isolats de M. tuberculosis ont été soumis aux tests de sensibilité aux antituberculeux. De ce nombre, 16 étaient identifiés comme $M$. bovis Bacillus Calmette-Guerin et ont été exclus des analyses ultérieures. La majorité des isolats, soit 1311 (89,9\%), étaient sensibles à tous les antituberculeux de première intention, et les 148 (10,1\%) autres isolats étaient résistants à un ou à plusieurs médicaments. La plupart de ceux-ci étaient monorésistants $(81,8 \%, \mathrm{n}=121)$, la monorésistance à l'isoniazide étant le profil de résistance le plus signalé $(n=89)$, suivie de la monorésistance au pyrazinamide $(n=26)$ et à la rifampicine $(n=6)$. La polyrésistance a été constatée dans cinq isolats qui ont tous démontré une résistance à la combinaison de l'isoniazide et du pyrazinamide. La tuberculose multirésistante a été détectée dans 21 isolats et la tuberculose ultrarésistante, dans un seul (figure 1).
Figure 1 : Isolats de tuberculose testés pour la sensibilité aux antituberculeux au Canada, 2018

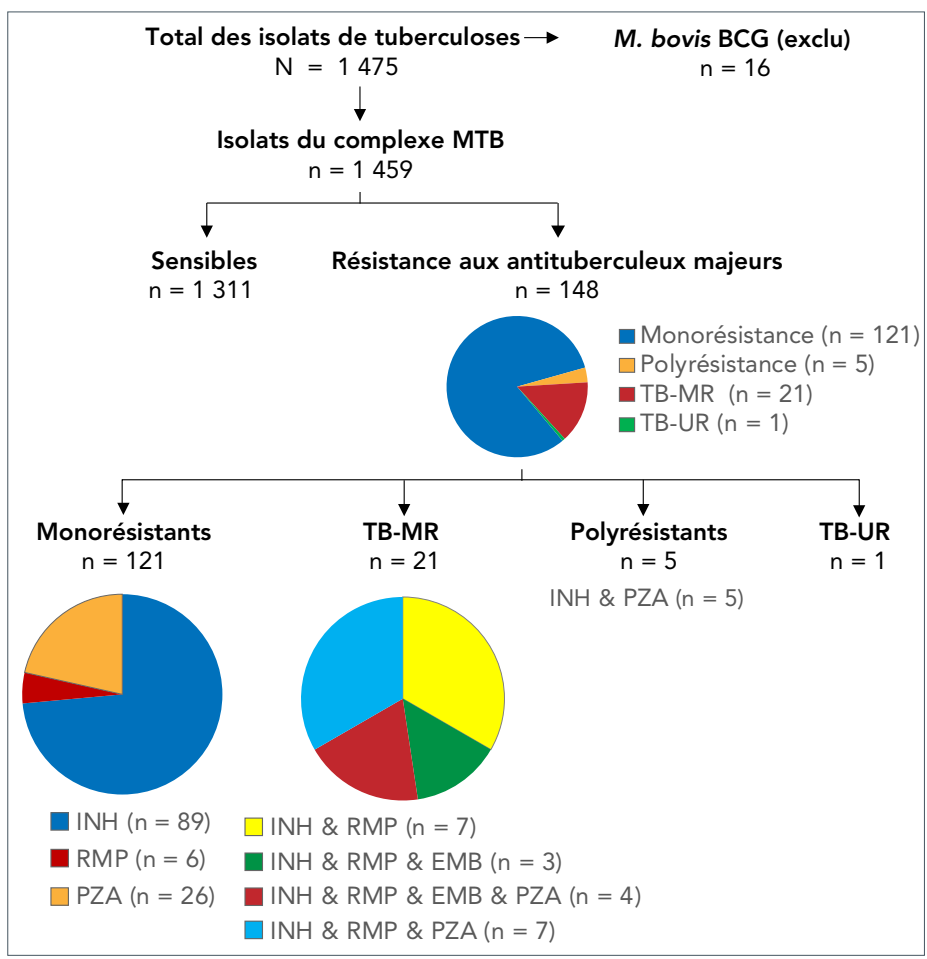

Abréviations : BCG, Bacillus Calmette-Guérin; EMB, éthambutol; INH, isoniazide MTB, Mycobacterium tuberculosis; M. bovis, Mycobacterium bovis; PZA, pyrazinamide; RMP, rifampicide; TB, tuberculose; TB-MR, tuberculose multirésistante; TB-UR, tuberculose ultrarésistante

Entre 2008 et 2018, la proportion d'isolats démontrant une pharmaco-résistance aux antituberculeux a connu peu de changement au Canada (figure 2). Elle a fluctué au cours des ans, mais est restée en dedans d'une marge étroite ( $8,1 \%$ à 10,5\%). Au cours de cette période, la proportion de la monorésistance oscillait entre $6,8 \%$ et $9,1 \%$; celle de la polyrésistance, entre $0,0 \%$ et $0,8 \%$; celle de la multirésistance, entre $0,6 \%$ et $1,6 \%$;

Figure 2 : Proportion des isolats de tuberculose déclarés présentant une résistance aux antituberculeux, par profil de résistance aux antituberculeux, Canada, 2008 à 2018

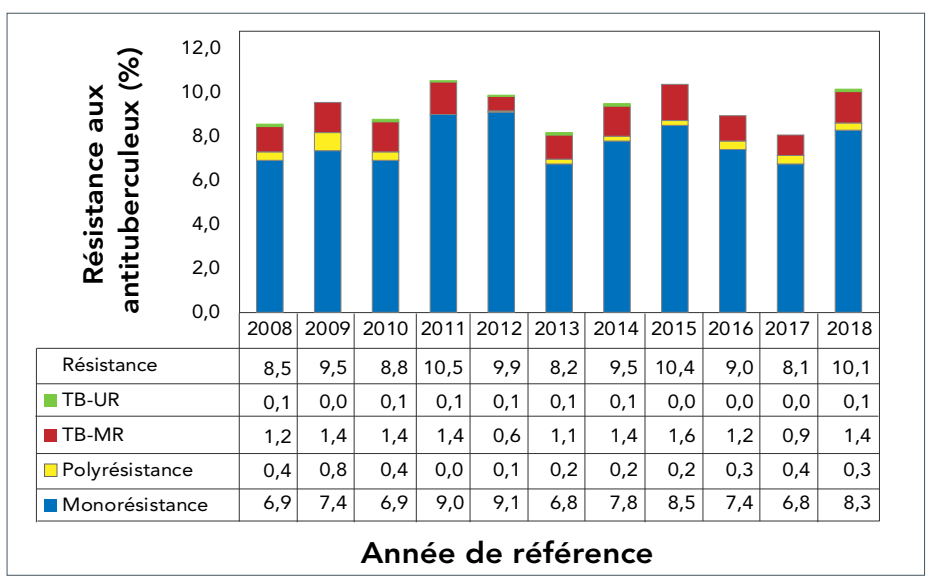

Abréviations : TB-MR, tuberculose multirésistante; TB-UR, tuberculose ultrarésistante 
et celle de l'ultrarésistance,entre $0,0 \%$ et $0,1 \%$. Au cours de cette période, un total de sept isolats de tuberculose ultrarésistante ont été signalés, jamais plus d'un isolat par année (figure 2).

\section{Distribution géographique des cas de TB résistants aux antituberculeux}

En 2018, la plupart des isolats soumis aux tests de sensibilité aux antituberculeux provenaient de l'Ontario $(n=546$; $37,4 \%$ ), suivi de la Colombie-Britannique ( $n=255 ; 17,5 \%)$, du Québec ( $n=213 ; 14,6 \%$ ), de l'Alberta ( $n=161 ; 11,0 \%$ ) et du Manitoba ( $n=149 ; 10,2 \%$ ). Les provinces de l'Atlantique (Terre-Neuve-et-Labrador, I'Île-du-Prince-Édouard, la Nouvelle-Écosse et le Nouveau- Brunswick) ainsi que les territoires (le Yukon, les Territoires-du-Nord-Ouest et le Nunavut) ont déclaré entre zéro et deux isolats présentant chacun une résistance quelconque aux antituberculeux (tableau 2).

Le nombre le plus élevé d'isolats a été enregistré en Ontario ( $n=68)$, au Québec $(n=25)$ et en Colombie-Britannique $(n=23)$, qui ensemble représentaient environ $78,4 \%(n=116)$ des isolats pharmacorésistants signalés (tableau 2). La résistance à tout antituberculeux était légèrement supérieure à la moyenne $(10,1 \%)$ en Saskatchewan $(13,6 \%)$, en Ontario (12,5\%) et au Québec (11,7\%), et inférieure en Colombie-Britannique $(9,0 \%)$, au Manitoba (6,7 \%), à Terre-Neuve-et-Labrador (5,6\%), en Alberta $(5,6 \%)$ et au Nunavut (2,7\%). Aucune résistance aux médicaments n'a été signalée à l'île-du-Prince-Édouard, les Territoires-du-Nord-Ouest, ni dans le Yukon.
Quant aux isolats ayant présenté une tuberculose multirésistante en 2018, I'Ontario en a déclaré le plus grand nombre $(n=13)$, suivi de l'Alberta $(n=3)$ et de la Colombie-Britannique $(n=2)$. Le Nouveau-Brunswick, le Québec et le Manitoba ont chacun déclaré un seul isolat de tuberculose multirésistante. Le seul isolat de tuberculose ultrarésistante déclaré en 2018 a été signalé en Saskatchewan.

\section{Cas de résistance aux antituberculeux par groupe d'âge}

Parmi tous les isolats éligibles soumis aux tests de sensibilité aux antituberculeux en 2018 ( $n=1459)$, seulement 1,9\% ( $n=27)$ provenait de personnes âgées de moins de 15 ans. La répartition des isolats dans les autres groupes d'âge variait entre 11,2\% $(n=163)$ chez les 55 à 64 ans et $18,4 \%(n=268)$ chez les 25 à 34 ans.

La proportion d'isolats présentant une résistance quelconque aux antituberculeux était de $7,4 \%(n=2)$ chez les personnes âgées de moins de 15 ans; cependant, aucun de ces isolats n'était multirésistant. Chez les personnes de plus de 14 ans, la proportion d'isolats présentant une résistance quelconque aux antituberculeux variait entre $8,2 \%(n=208)$ dans le groupe de 15 à 24 ans d'âge et 11,8\% dans les tranches d'âge de 35 à 44 ans $(n=211)$ et de 65 à 74 ans $(n=169)$. La proportion représentant la tuberculose multirésistante (figure 3 ) variait de $0,8 \%(n=2)$ chez les individus de 75 ans et plus à $1.9 \%(n=4)$ chez les individus âgés de 15 à 24 ans et de 35 à 44 ans.

Tableau 2 : Nombre et proportion d'isolats présentant une résistance aux antituberculeux, par province ou territoire, Canada, 2018

\begin{tabular}{|c|c|c|c|c|c|c|c|c|c|c|c|c|}
\hline \multirow{2}{*}{ PT } & \multicolumn{2}{|c|}{ Total des isolats } & \multicolumn{2}{|c|}{ Résistance } & \multicolumn{2}{|c|}{ Monorésistance } & \multicolumn{2}{|c|}{ Polyrésistance } & \multicolumn{2}{|c|}{ TB-MR } & \multicolumn{2}{|c|}{ TB-UR } \\
\hline & n & $\%$ & $\mathrm{n}$ & $\%$ & n & $\%$ & n & $\%$ & $\mathbf{n}$ & $\%$ & $n$ & $\%$ \\
\hline T.-N.-L. & 18 & 1,2 & 1 & 5,6 & 1 & 5,6 & 0 & 0,0 & 0 & 0,0 & 0 & 0,0 \\
\hline Î.-P.-É. & 2 & 0,1 & 0 & 0,0 & 0 & 0,0 & 0 & 0,0 & 0 & 0,0 & 0 & 0,0 \\
\hline N.-É. & 8 & 0,5 & 2 & 25,0 & 2 & 25,0 & 0 & 0,0 & 0 & 0,0 & 0 & 0,0 \\
\hline N.-B. & 6 & 0,4 & 1 & 16,7 & 0 & 0,0 & 0 & 0,0 & 1 & 16,7 & 0 & 0,0 \\
\hline Qc & 213 & 14,6 & 25 & 11,7 & 23 & 10,8 & 1 & 0,5 & 1 & 0,5 & 0 & 0,0 \\
\hline Ont. & 546 & 37,4 & 68 & 12,5 & 54 & 9,9 & 1 & 0,2 & 13 & 2,4 & 0 & 0,0 \\
\hline Man. & 149 & 10,2 & 10 & 6,7 & 9 & 6,0 & 0 & 0,0 & 1 & 0,7 & 0 & 0,0 \\
\hline Sask. & 59 & 4,0 & 8 & 13,6 & 6 & 10,2 & 1 & 1,7 & 0 & 0,0 & 1 & 1,7 \\
\hline Alb. & 161 & 11,0 & 9 & 5,6 & 5 & 3,1 & 1 & 0,6 & 3 & 1,9 & 0 & 0,0 \\
\hline C.-B. & 255 & 17,5 & 23 & 9,0 & 20 & 7,8 & 1 & 0,4 & 2 & 0,8 & 0 & 0,0 \\
\hline Yn & 3 & 0,2 & 0 & 0,0 & 0 & 0,0 & 0 & 0,0 & 0 & 0,0 & 0 & 0,0 \\
\hline T.N.-O. & 3 & 0,2 & 0 & 0,0 & 0 & 0,0 & 0 & 0,0 & 0 & 0,0 & 0 & 0,0 \\
\hline $\mathrm{Nt}$ & 36 & 2,5 & 1 & 2,8 & 1 & 2,8 & 0 & 0,0 & 0 & 0,0 & 0 & 0,0 \\
\hline Total & 1459 & 100,0 & 148 & 10,1 & 121 & 8,3 & 5 & 0,3 & 21 & 1,4 & 1 & 0,1 \\
\hline
\end{tabular}

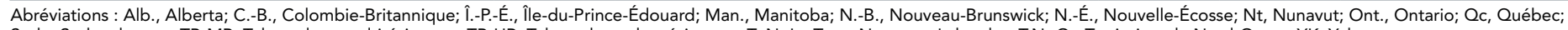
Sask., Saskatchewan; TB-MR, Tuberculose multirésistante; TB-UR, Tuberculose ultrarésistante; T.-N.-L., Terre-Neuve-et-Labrador; T.N.-O., Territoires du Nord-Ouest; YK, Yukon 
Figure 3 : Nombre et proportion des isolats de tuberculose déclarés présentant une résistance aux antituberculeux, par groupe d'âge et profil de résistance, Canada, 2018

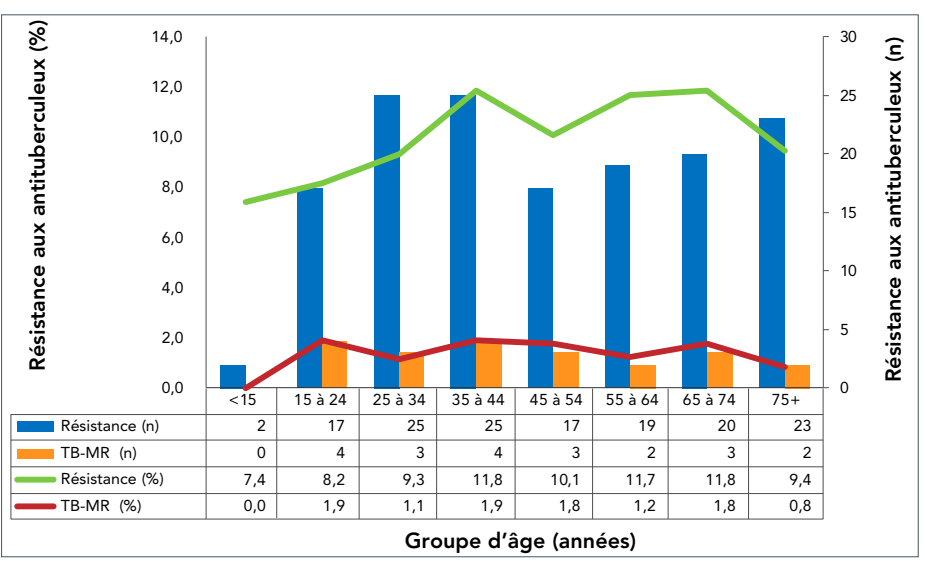

Abréviations : TB-MR, tuberculose multirésistante; $<$, plus jeune que

\section{Résistance aux antituberculeux par sexe}

Parmi les isolats soumis aux tests de sensibilité aux antituberculeux en 2018, ceux venant des hommes représentaient $54,9 \%(n=801)$ et ceux des femmes, $45,0 \%$ $(n=657)$. Le sexe du cas n'a pas été déclaré pour un isolat. Les proportions d'isolats présentant une résistance aux antituberculeux étaient similaires pour les deux sexes. Chez les hommes, 9,6\% $(n=77)$ des isolats de tuberculose ont présenté une résistance quelconque aux antituberculeux de première intention et $1,4 \%(n=11)$ étaient des isolats de tuberculose multirésistante. Chez les femmes, $10,8 \%(n=71)$ des isolats présentaient une résistance quelconque, et 10 (1,5\%) étaient des isolats de tuberculose multirésistante. Ces résultats sont cohérents avec la tendance des cinq années précédentes (2013 à 2017), où les proportions d'une résistance quelconque étaient plus élevées chez les femmes que chez les hommes (figure 4).

\section{Résistance aux antituberculeux par pays de naissance}

De 2006 à 2016, les isolats venant des Canadiens nés à l'étranger ont exprimé le niveau élevé de pharmaco-résistance aux antituberculeux ( $n=1086$ de 10110 isolats; 10,7\%) comparativement à ceux des Canadiens nés au Canada ( $n=211$ de 4373 isolats; 4,8\%). Les proportions des isolats de tuberculose multirésistante suivaient une tendance similaire, soit $1,4 \%(n=148)$ chez les Canadiens nés à l'étranger par rapport à $0,2 \%(n=7)$ pour ceux nés au Canada. Certaines différences dans les proportions de pharmacorésistance ont également été détectées parmi différents sous-groupes de cas nés au Canada. De 2006 à 2016, 2,4 \% ( $n=68$ de 2822 isolats) des cas de tuberculose chez les Autochtones nés au Canada étaient résistants à au moins un antituberculeux de première intention. II n'y avait aucun cas de tuberculose multirésistante dans ce groupe. Parmi les isolats échantillonnés chez les cas nonAutochtones nés au Canada, 9,3\% ( $n=145$ de 1551 isolats) ont exprimé une résistance à au moins un antituberculeux; et parmi ces derniers, $4,8 \%(n=7)$ étaient des cas de tuberculose multirésistante.

Figure 4 : Pourcentage des isolats de tuberculose déclarés présentant une résistance aux antituberculeux, par sexe et profil de résistance, Canada, 2008 à 2018

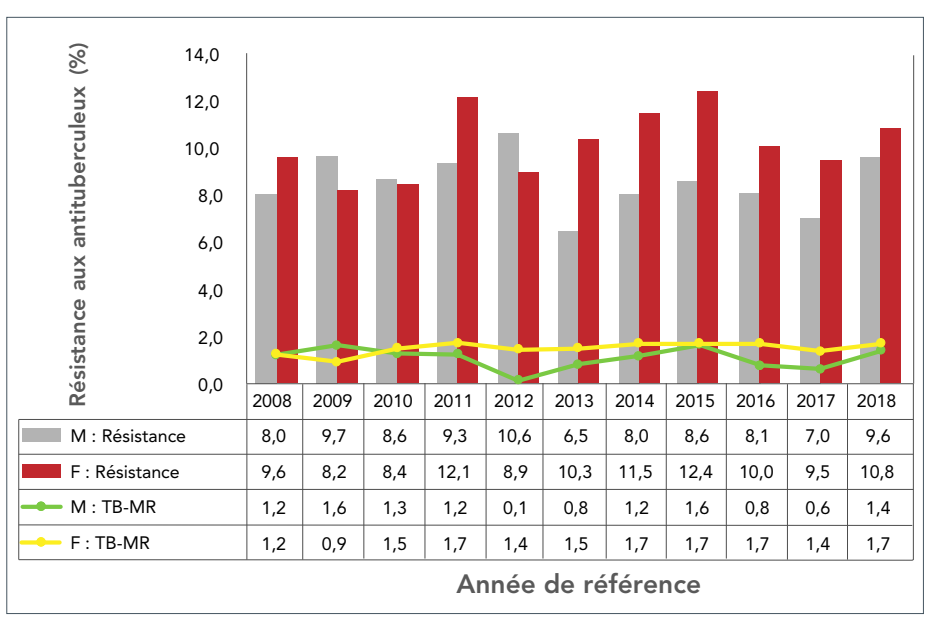

Abréviations : $\mathrm{F}$, femme; $\mathrm{H}$, homme; TB-MR, tuberculose multirésistante

\section{Discussion}

En 2018, la proportion de cas de résistance aux antituberculeux parmi les isolats de tuberculose à culture positive au Canada est restée faible. Même si le pourcentage d'isolats présentant une résistance quelconque aux antituberculeux a augmenté de 8,1\% en 2017 à 10,1\% en 2018, ce pourcentage est resté dans les limites des proportions observées ( $8,1 \%$ à $10,5 \%$ ) au cours des 10 dernières années. Aucune tendance nouvelle n'a été décelée par rapport à l'âge, le sexe ou au pays de naissance. Signalons par ailleurs qu'un seul isolat de tuberculose ultrarésistante a été déclaré au Canada en 2018. Même s'il s'agit là d'un premier isolat de ce genre signalé depuis 2014, il n'est pas inhabituel d'en voir un de temps en temps, puisqu'un isolat de tuberculose ultrarésistante par année a été déclaré au courant des six différentes années de la dernière décennie. En 2017, I'OMS estimait qu'environ $3,5 \%$ des nouveaux cas de tuberculose à l'échelle internationale présentaient une résistance à la rifampicine ou étaient des cas de tuberculose multirésistante (2). Les données Canadiennes sont significativement très en deçà de ces estimations comme le montrent les résultats de 2018 qui recensent juste $1,8 \%$ des isolats avec une résistance à la rifampicine ou une multirésistance.

\section{Limites}

Le SCSLT est le résultat d'une collaboration entre les gouvernements fédéral, provinciaux et territoriaux et les laboratoires de santé publique. En tant que principale source de données nationales sur la résistance aux antituberculeux au Canada, les données contenues dans le présent article ont pour but d'éclairer les mesures de santé publique, ainsi que l'élaboration et l'évaluation des politiques et des programmes. 
Toutefois, certaines limites doivent entre prises en compte lors de l'interprétation de présents résultats. II est important de rappeler que puisque le SCSLT est un système de surveillance en laboratoire, les données démographiques disponibles sont limitées, et les isolats déclarés ne peuvent pas être directement reliés aux données de surveillance basées sur des cas provenant du SCDTB. Il a été démontré que les informations sur la résistance aux antituberculeux déclarées dans le SCDTB sont assez exhaustives (8) et raisonnablement comparables à celles du SCSLT, malgré l'existence possible de certaines divergences irréconciliables. Parmi les efforts futurs visant à améliorer la surveillance de la tuberculose, on examine la possibilité d'intégrer les deux systèmes de surveillance de façon à fournir des données épidémiologiques détaillées sur les cas de tuberculose pharmaco-résistante.

Même si, à l'échelle mondiale et au Canada, le nombre total de cas de tuberculose chez les enfants de moins de 15 ans est faible (9), il est possible que les données contenues dans le présent article sous-représentent la proportion de cas de tuberculose pharmaco-résistante dans ce groupe d'âge, car il est difficile d'obtenir des prélèvements d'expectoration chez les jeunes enfants pour des tests de sensibilité aux antituberculeux à base de culture.

\section{Conclusion}

En 2018, la proportion des isolats présentant une résistance aux antituberculeux au Canada est restée relativement stable et inférieure aux moyennes internationales, tant démographiquement que géographiquement.

\section{Déclaration des auteurs}

M. L. - Conceptualisation, méthodologie, logiciels, validation, analyse formelle et rédaction (ébauche originale)

D. D. - Conceptualisation, logiciels, validation, analyse formelle, rédaction (examen) et révision, visualisation

L. S. - Validation, rédaction (examen) et révision

S. M. - Conceptualisation, rédaction (examen) et révision, supervision

\section{Conflit d'intérêts}

Aucun.

\section{Collaborateurs}

La Division de surveillance de la santé et de l'épidémiologie du Centre de lutte contre les maladies transmissibles et les infections de l'Agence de la santé publique du Canada tient à remercier les équipes du Réseau technique canadien de laboratoires de tuberculose pour leur contribution et leur participation au Système canadien de surveillance des laboratoires de tuberculose (SCSLT) :
Membres du Réseau technique canadien de laboratoires de tuberculose: H. Adam, P. M. Akochy, R. Bittner, K. Cronin, D. Farrell, D. Haldane, H. Hannah, F. Jamieson, H. Mackenzie, E. Martin, R. Needle, K. Ray, M. Rodrigues, I. Sekirov, C. Shandro, H. Soualhine, R. Thomas, G. Tyrrell.

\section{Financement}

Ce travail a été réalisé grâce au soutien de l'Agence de la santé publique du Canada, dans le cadre de son mandat de base.

\section{Références}

1. World Health Organization. Global tuberculosis report 2018. Geneva (CH): World Health Organization; 2018.

2. Menzies $D$, Wong $T$, editeurs. Normes Canadiennes pour la lutte antituberculeuse 7e édi-tion. Ottawa (ON): Agence de la santé publique du Canada; 2014. Une publication cojointe avec la Société canadienne de thoracologie de l'Association pulmonaire du Canada. https://www.canada.ca/fr/sante-publique/services/maladiesinfectieuses/normes-canadiennes-lutte-antituberculeuse-7e-edition. html

3. Organisation mondiale de la Santé. UN General Assembly high-level meeting on ending TB: New York, 26 September 2018. Geneva (CH): World Health Organization; 2018. https://www.who.int/news-room/ events/un-general-assembly-high-level-meeting-on-ending-tb

4. United Nations. Political declaration of the high-level meeting of the United Nations General Assembly on the fight against tuberculosis: resolution / adopted by the General As-sembly. New York: United Nations ; 2018(A/RES/73/3).

5. LaFreniere $\mathrm{M}$, Hussain $\mathrm{H}$, Vachon $\mathrm{J}$. La résistance aux antituberculeux au Canada : 2017. Relevé des maladies transmissibles au Canada 2018; 44(11):330-6. DOI

6. Agence de la santé publique du Canada. La tuberculose : La résistance aux antituberculeux au Canada - 2015. Ottawa (ON): Ministre des Travaux publics et des Services gouverne-mentaux Canada; 2017. https://www.canada.ca/content/dam/phac-aspc/ documents/services/publications/diseases-conditions/tuberculosisdrug-resistance-canada-2015/tuberculose-resistance-auxantituberculeux-canada-2015.pdf

7. Agence de la santé publique du Canada. La tuberculose au Canada 2012. Ottawa (ON): Mi-nistre des Travaux publics et des Services gouvernementaux Canada; 2015. https://www.canada.ca/content/ dam/phac-aspc/migration/phac-aspc/tbpc-latb/pubs/tbcan12/ assets/pdf/tbcan12-fra.pdf

8. Agence de la santé publique du Canada. Système canadien de surveillance de la résistance aux antimicrobiens. Ottawa (ON) : Agence de la santé publique du Canada; 2018. https://www.canada. $\mathrm{ca/content/dam/phac-aspc/documents/services/publications/drugs-}$ health-products/canadian-antimicrobial-resistance-surveillancesystem-2017-report-executive-summary/SCSRA-Rapport-2017-Fr.pdf

9. LaFreniere $\mathrm{M}$, Hussain $\mathrm{H}, \mathrm{He} \mathrm{N}, \mathrm{McGuire} \mathrm{M}$. La tuberculose au Canada : 2017. Relevé des maladies transmissibles au Canada 2019;45(2/3):73-80. DOI 


\section{Annexe : Liste des tableaux supplémentaires disponibles sur demande}

Tableau supplémentaire 1 : Nombre total et pourcentage des isolats du complexe Mycobacterium tuberculosis identifiés comme présentant une résistance quelconque, une multirésistance et une ultrarésistance, par année, 2008 à 2018, Canada

Tableau supplémentaire 2 : Profil global de la résistance aux antituberculeux déclarée au Canada, 2008 à 2018

Tableau supplémentaire 3 : Résultats des épreuves systématiques de sensibilité aux antituberculeux des isolats du complexe Mycobacterium tuberculosis provenant de l'Alberta, 2008 à 2018

Tableau supplémentaire 4 : Résultats des épreuves systématiques de sensibilité aux antituberculeux des isolats du complexe Mycobacterium tuberculosis provenant de la ColombieBritannique, 2008 à 2018

Tableau supplémentaire 5 : Résultats des épreuves systématiques de sensibilité aux antituberculeux des isolats du complexe Mycobacterium tuberculosis provenant du Manitoba, 2008 à 2018

Tableau supplémentaire 6 : Résultats des épreuves systématiques de sensibilité aux antituberculeux des isolats du complexe Mycobacterium tuberculosis provenant du Nouveau-Brunswick, 2008 à 2018

Tableau supplémentaire 7 : Résultats des épreuves systématiques de sensibilité aux antituberculeux des isolats du complexe Mycobacterium tuberculosis provenant de Terre-Neuve-etLabrador, 2008 à 2018

Tableau supplémentaire 8 : Résultats des épreuves systématiques de sensibilité aux antituberculeux des isolats du complexe Mycobacterium tuberculosis provenant des Territoires-du-Nord-

Ouest, 2008 à 2018

Tableau supplémentaire 9 : Résultats des épreuves systématiques de sensibilité aux antituberculeux des isolats du complexe Mycobacterium tuberculosis provenant de la Nouvelle-Écosse, 2008 à 2018

Tableau supplémentaire 10 : Résultats des épreuves systématiques de sensibilité aux antituberculeux des isolats du complexe Mycobacterium tuberculosis provenant du Nunavut, 2008 à 2018

Tableau supplémentaire 11 : Résultats des épreuves systématiques de sensibilité aux antituberculeux des isolats du complexe Mycobacterium tuberculosis provenant de l'Ontario, 2008 à 2018

Tableau supplémentaire 12 : Résultats des épreuves systématiques de sensibilité aux antituberculeux des isolats du complexe Mycobacterium tuberculosis provenant de l'île-duPrince-Édouard, 2008 à 2018

Tableau supplémentaire 13 : Résultats des épreuves systématiques de sensibilité aux antituberculeux des isolats du complexe Mycobacterium tuberculosis provenant du Québec, 2008 à 2018

Tableau supplémentaire 14 : Résultats des épreuves systématiques de sensibilité aux antituberculeux des isolats du complexe Mycobacterium tuberculosis provenant de la Saskatchewan, 2008 à 2018

Tableau supplémentaire 15 : Résultats des épreuves systématiques de sensibilité aux antituberculeux des isolats du complexe Mycobacterium tuberculosis provenant du Yukon, 2008 à 2018

Tableau supplémentaire 16 : Isolats de tuberculose multirésistante et de tuberculose ultrarésistante par province ou territoire d'origine, 2018

Tableau supplémentaire 17 : Nombre total d'isolats du complexe Mycobacterium tuberculosis par province ou territoire de déclaration et d'origine, 2018

Tableau supplémentaire 18 : Répartition entre les provinces et territoires par tuberculose présentant une résistance quelconque, multirésistante et ultrarésistante au Canada, 2008 à 2018

Tableau supplémentaire 19 : Résistance aux antituberculeux par sexe et groupe d'âge au Canada, 2018 